Pacific

Journal of

Mathematics

\title{
A SIMPLE SOLUTION TO THE WORD PROBLEM FOR VIRTUAL BRAID GROUPS
}

Paolo Bellingeri, Bruno A. Cisneros de la Cruz and Luis Paris 


\title{
A SIMPLE SOLUTION TO THE WORD PROBLEM FOR VIRTUAL BRAID GROUPS
}

\author{
Paolo Bellingeri, Bruno A. Cisneros de la CRuz And Luis Paris
}

We show a simple and easily implementable solution to the word problem for virtual braid groups.

\section{Introduction}

Virtual braid groups were introduced by L. Kauffman [1999] in his seminal paper on virtual knots and links. They can be defined in several ways, such as in terms of Gauss diagrams [Bar-Natan and Dancso 2015; Cisneros de la Cruz 2015], in terms of braids in thickened surfaces [Cisneros de la Cruz 2015], and in terms of virtual braid diagrams. The latter will be our starting point of view.

A virtual braid diagram on $n$ strands is an $n$-tuple $\beta=\left(b_{1}, \ldots, b_{n}\right)$ of smooth paths in the plane $\mathbb{R}^{2}$ satisfying the following conditions:

(a) $b_{i}(0)=(i, 0)$ for all $i \in\{1, \ldots, n\}$.

(b) There is a permutation $g \in \mathfrak{S}_{n}$ such that $b_{i}(1)=(g(i), 1)$ for all $i \in\{1, \ldots, n\}$.

(c) $\left(p_{2} \circ b_{i}\right)(t)=t$ for all $i \in\{1, \ldots, n\}$ and all $t \in[0,1]$, where $p_{2}: \mathbb{R}^{2} \rightarrow \mathbb{R}$ denotes the projection on the second coordinate.

(d) The $b_{i}$ intersect transversely in a finite number of double points, called the crossings of the diagram.

Each crossing is endowed with one of the following attributes: positive, negative, virtual. In the figures they are generally indicated as in Figure 1 . Let $\mathrm{VBD}_{n}$ be the set of virtual braid diagrams on $n$ strands, and let $\sim$ be the equivalence relation on $\mathrm{VBD}_{n}$ generated by ambient isotopy and the virtual Reidemeister moves depicted in Figure 2. The concatenation of diagrams induces a group structure on $\mathrm{VBD}_{n} / \sim$. The latter is called the virtual braid group on $n$ strands, and is denoted by $\mathrm{VB}_{n}$.

MSC2010: primary 20F36; secondary 20F10, 57M25.

Keywords: word problem, virtual braid group. 


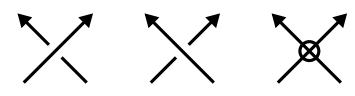

Figure 1. Positive, negative and virtual crossings in a virtual braid diagram.

It was observed in [Kamada 2007; Vershinin 2001] that $\mathrm{VB}_{n}$ has a presentation with generators $\sigma_{1}, \ldots, \sigma_{n-1}, \tau_{1}, \ldots, \tau_{n-1}$ and relations

$$
\begin{aligned}
\tau_{i}^{2} & =1 & & \text { for } 1 \leq i \leq n-1, \\
\sigma_{i} \sigma_{j}=\sigma_{j} \sigma_{i}, \sigma_{i} \tau_{j} & =\tau_{j} \sigma_{i}, \quad \tau_{i} \tau_{j}=\tau_{j} \tau_{i} & & \text { for }|i-j| \geq 2, \\
\sigma_{i} \sigma_{j} \sigma_{i}=\sigma_{j} \sigma_{i} \sigma_{j}, \quad \sigma_{i} \tau_{j} \tau_{i} & =\tau_{j} \tau_{i} \sigma_{j}, \quad \tau_{i} \tau_{j} \tau_{i}=\tau_{j} \tau_{i} \tau_{j} & & \text { for }|i-j|=1 .
\end{aligned}
$$

A solution to the word problem for virtual braid groups was shown in [Godelle and Paris 2012]. However, this solution is quite theoretical and its understanding requires some heavy technical knowledge on Artin groups. Therefore, it is incomprehensible and useless for most of the potential users, including low-dimensional topologists. Moreover, its implementation would be difficult. Our aim here is to show a new solution, which is simpler and easily implementable, and whose understanding does not require any special technical knowledge. This new solution is in the spirit of the one shown in [Godelle and Paris 2012], in the sense that one of the main ingredients in its proof is the study of parabolic subgroups in Artin groups.

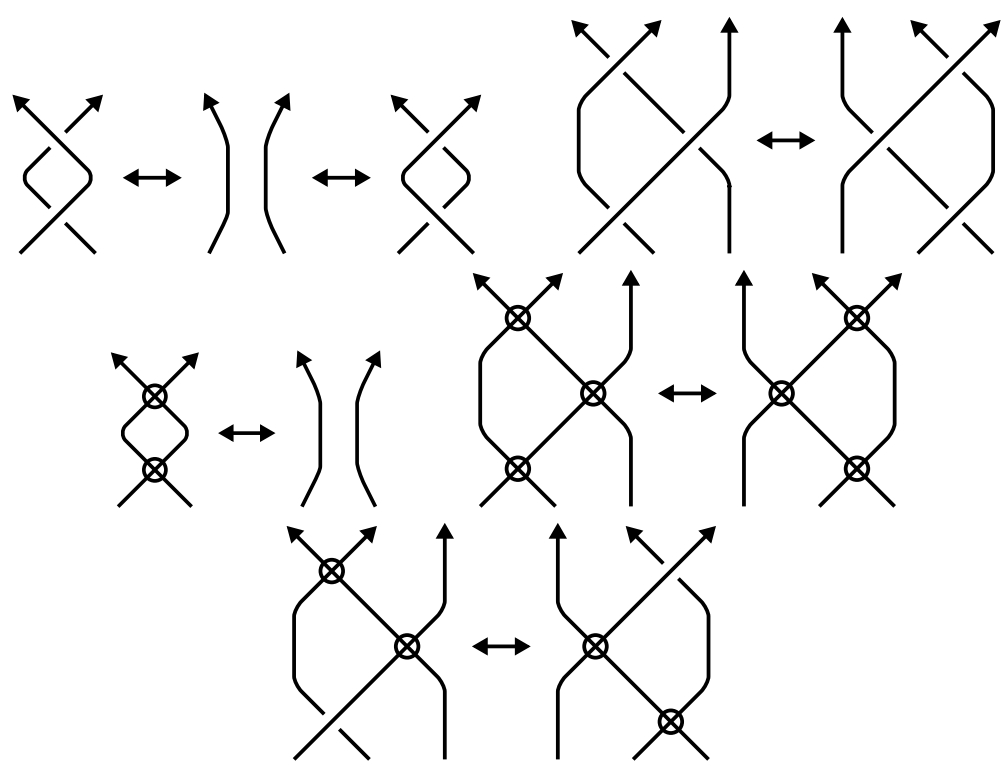

Figure 2. Virtual Reidemeister moves. 
We have not calculated the complexity of this algorithm, as this is probably at least exponential because of the inductive step 3 (see Section 2). Nevertheless, it is quite efficient for a limited number of strands (see the example at the end of Section 2), and, above all, it should be useful to study theoretical questions on $\mathrm{VB}_{n}$ such as the faithfulness of representations of this group in automorphism groups of free groups and/or in linear groups. Note that the faithfulness of such a representation will immediately provide another, probably faster, solution to the word problem for $\mathrm{VB}_{n}$.

The Burau representation easily extends to $\mathrm{VB}_{n}$ [Vershinin 2001], but the question whether $\mathrm{VB}_{n}$ is linear or not is still open. A representation of $\mathrm{VB}_{n}$ in $\operatorname{Aut}\left(F_{n+1}\right)$ was independently constructed in [Bardakov 2005] and [Manturov 2003], but such a representation has recently been proven to be not faithful for $n \geq 4$ [Chterental 2015, Proposition 5.3] (see the example at the end of Step 1). So, we do not know yet any representation on which we can test our algorithm.

Chterental [2015] shows a faithful action of $\mathrm{VB}_{n}$ on a set of objects that he calls "virtual curve diagrams". We have some hope to use this action to describe another explicit solution to the word problem for $\mathrm{VB}_{n}$. But, for now, we do not know any formal definition of this action, nor how it could be encoded in an algorithm.

\section{The algorithm}

Our solution to the word problem for $\mathrm{VB}_{n}$ is divided into four steps. In Step 1 we define a subgroup $\mathrm{KB}_{n}$ of $\mathrm{VB}_{n}$ and a generating set $\mathcal{S}$ for $\mathrm{KB}_{n}$, and we show an algorithm (called Algorithm A) which decides whether an element of $\mathrm{VB}_{n}$ belongs to $\mathrm{KB}_{n}$ and, if yes, determines a word over $\mathcal{S}^{ \pm 1}$ which represents this element. For $\mathcal{X} \subset \mathcal{S}$, we denote by $\mathrm{KB}_{n}(\mathcal{X})$ the subgroup of $\mathrm{KB}_{n}$ generated by $\mathcal{X}$. The other three steps provide a solution to the word problem for $\mathrm{KB}_{n}(\mathcal{X})$ which depends recursively on the cardinality of $\mathcal{X}$. Step 2 is the beginning of the induction. More precisely, the algorithm proposed in Step 2 (called Algorithm B) is a solution to the word problem for $\mathrm{KB}_{n}(\mathcal{X})$ when $\mathcal{X}$ is a full subset of $\mathcal{S}$ (the notion of "full subset" will be also defined in Step 2; for now, the reader just need to know that singletons are full subsets). In Step 3 we suppose given a solution to the word problem for $\mathrm{KB}_{n}(\mathcal{X})$, and, for a given subset $\mathcal{Y} \subset \mathcal{X}$, we show an algorithm which solves the membership problem for $\mathrm{KB}_{n}(\mathcal{Y})$ in $\mathrm{KB}_{n}(\mathcal{X})$ (called Algorithm $\mathrm{C}$ ). In Step 4 we show an algorithm which solves the word problem for $\operatorname{KB}_{n}(\mathcal{X})$ when $\mathcal{X}$ is not a full subset, under the assumption that the group $\operatorname{KB}_{n}(\mathcal{Y})$ has a solvable word problem for any proper subset $\mathcal{Y}$ of $\mathcal{X}$ (called Algorithm D).

Step 1. Recall that $\mathfrak{S}_{n}$ denotes the group of permutations of $\{1, \ldots, n\}$. We denote by $\theta: \mathrm{VB}_{n} \rightarrow \mathfrak{S}_{n}$ the epimorphism which sends $\sigma_{i}$ to 1 and $\tau_{i}$ to $(i, i+1)$ for all $1 \leq i \leq n-1$, and by $\mathrm{KB}_{n}$ the kernel of $\theta$. Note that $\theta$ has a section $\iota: \mathfrak{S}_{n} \rightarrow \operatorname{VB}_{n}$ 

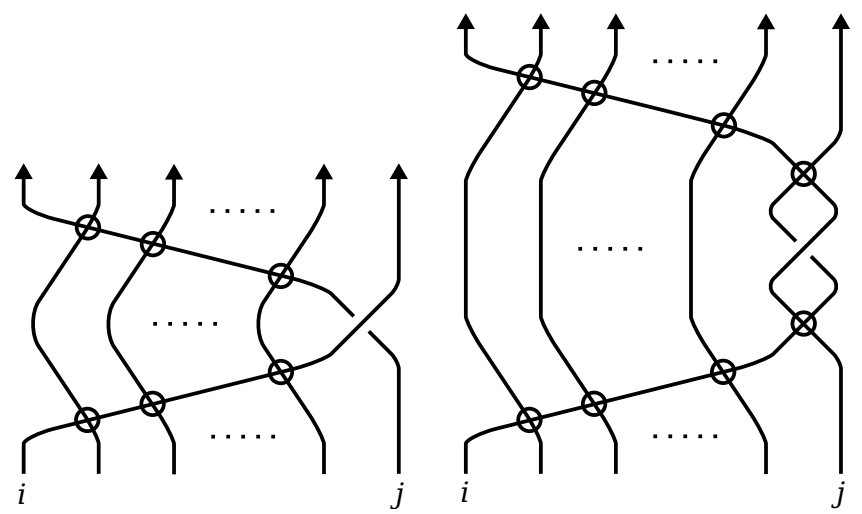

Figure 3. Generators for $\mathrm{KB}_{n}: \delta_{i, j}$ (left) and $\delta_{j, i}$ (right)

which sends $(i, i+1)$ to $\tau_{i}$ for all $1 \leq i \leq n-1$, and therefore $\mathrm{VB}_{n}$ is a semidirect product $\mathrm{VB}_{n}=\mathrm{KB}_{n} \rtimes \mathfrak{S}_{n}$. The following proposition is proved in Rabenda's master's thesis, which, unfortunately, is not available anywhere. However, its proof can also be found in [Bardakov and Bellingeri 2009].

Proposition 2.1. For $1 \leq i<j \leq n$ we set

$$
\begin{aligned}
& \delta_{i, j}=\tau_{i} \tau_{i+1} \cdots \tau_{j-2} \sigma_{j-1} \tau_{j-2} \cdots \tau_{i+1} \tau_{i}, \\
& \delta_{j, i}=\tau_{i} \tau_{i+1} \cdots \tau_{j-2} \tau_{j-1} \sigma_{j-1} \tau_{j-1} \tau_{j-2} \cdots \tau_{i+1} \tau_{i} .
\end{aligned}
$$

Then $\mathrm{KB}_{n}$ has a presentation with generating set

$$
\mathcal{S}=\left\{\delta_{i, j} \mid 1 \leq i \neq j \leq n\right\}
$$

and relations

$$
\begin{aligned}
\delta_{i, j} \delta_{k, l} & =\delta_{k, l} \delta_{i, j} & & \text { for } i, j, k, l \text { distinct }, \\
\delta_{i, j} \delta_{j, k} \delta_{i, j} & =\delta_{j, k} \delta_{i, j} \delta_{j, k} & & \text { for } i, j, k \text { distinct. }
\end{aligned}
$$

The virtual braids $\delta_{i, j}$ and $\delta_{j, i}$ are depicted in Figure 3.

The following is an important tool in the forthcoming Algorithm A.

Lemma 2.2 [Bardakov and Bellingeri 2009]. Let $u$ be a word over $\left\{\tau_{1}, \ldots, \tau_{n-1}\right\}$, let $\bar{u}$ be the element of $\mathrm{VB}_{n}$ represented by $u$, and let $i, j \in\{1, \ldots, n\}, i \neq j$. Then $\bar{u} \delta_{i, j} \bar{u}^{-1}=\delta_{i^{\prime}, j^{\prime}}$, where $i^{\prime}=\theta(\bar{u})(i)$ and $j^{\prime}=\theta(\bar{u})(j)$.

Note that $\tau_{i}^{-1}=\tau_{i}$, since $\tau_{i}^{2}=1$, for all $i \in\{1, \ldots, n-1\}$. Hence, the letters $\tau_{1}^{-1}, \ldots, \tau_{n-1}^{-1}$ are not needed in the above lemma and below.

We give an algorithm which, given a word $u$ over $\left\{\sigma_{1}^{ \pm 1}, \ldots, \sigma_{n-1}^{ \pm 1}, \tau_{1}, \ldots, \tau_{n-1}\right\}$, decides whether the element $\bar{u}$ of $\mathrm{VB}_{n}$ represented by $u$ belongs to $\mathrm{KB}_{n}$. If yes, it also determines a word $u^{\prime}$ over $\mathcal{S}^{ \pm 1}=\left\{\delta_{i, j}^{ \pm} \mid 1 \leq i \neq j \leq n\right\}$ which represents $\bar{u}$. The fact that this algorithm is correct follows from Lemma 2.2. 
Algorithm A. Let $u$ be a word over $\left\{\sigma_{1}^{ \pm 1}, \ldots, \sigma_{n-1}^{ \pm 1}, \tau_{1}, \ldots, \tau_{n-1}\right\}$. We write $u$ in the form

$$
u=v_{0} \sigma_{i_{1}}^{\varepsilon_{1}} v_{1} \cdots v_{l-1} \sigma_{i_{l}}^{\varepsilon_{l}} v_{l},
$$

where $v_{0}, v_{1}, \ldots, v_{l}$ are words over $\left\{\tau_{1}, \ldots, \tau_{n-1}\right\}$, and $\varepsilon_{1}, \ldots, \varepsilon_{l} \in\{ \pm 1\}$. On the other hand, for a word $v=\tau_{j_{1}} \cdots \tau_{j_{k}}$ over $\left\{\tau_{1}, \ldots, \tau_{n-1}\right\}$, we set $\theta(v)=$ $\left(j_{1}, j_{1}+1\right) \cdots\left(j_{k}, j_{k}+1\right) \in \mathfrak{S}_{n}$. Note that $\theta(\bar{u})=\theta\left(v_{0}\right) \theta\left(v_{1}\right) \cdots \theta\left(v_{l}\right)$. If $\theta(\bar{u}) \neq 1$, then $\bar{u} \notin \mathrm{KB}_{n}$. If $\theta(\bar{u})=1$, then $\bar{u} \in \mathrm{KB}_{n}$, and $\bar{u}$ is represented by

$$
u^{\prime}=\delta_{a_{1}, b_{1}}^{\varepsilon_{1}} \delta_{a_{2}, b_{2}}^{\varepsilon_{2}} \cdots \delta_{a_{l}, b_{l}}^{\varepsilon_{l}},
$$

where

$$
a_{k}=\theta\left(v_{0} \cdots v_{k-1}\right)\left(i_{k}\right) \quad \text { and } \quad b_{k}=\theta\left(v_{0} \cdots v_{k-1}\right)\left(i_{k}+1\right)
$$

for all $k \in\{1, \ldots, l\}$.

Example. Chterental [2015] proved that the Bardakov-Manturov representation of $\mathrm{VB}_{n}$ in $\operatorname{Aut}\left(F_{n+1}\right)$ (see for instance [Bardakov 2005] for the definition) is not faithful, showing that the element $\omega=\left(\tau_{3} \sigma_{2} \tau_{1} \sigma_{2}^{-1}\right)^{3}$ is nontrivial in $\mathrm{VB}_{4}$ while the corresponding automorphism of $F_{5}$ is trivial. In [Chterental 2015] the nontriviality of $\omega$ is shown by means of an action on some curve diagrams, but this fact can easily be checked with Algorithm A. Indeed, $\theta(\omega)=((3,4)(1,2))^{3}=(3,4)(1,2) \neq 1$, hence $\omega \neq 1$.

Step 2. Let $S$ be a finite set. A Coxeter matrix over $S$ is a square matrix $M=$ $\left(m_{s, t}\right)_{s, t \in S}$, indexed by the elements of $S$, such that $m_{s, s}=1$ for all $s \in S$, and $m_{s, t}=m_{t, s} \in\{2,3,4, \ldots\} \cup\{\infty\}$ for all $s, t \in S, s \neq t$. We represent this Coxeter matrix with a labeled graph $\Gamma=\Gamma_{M}$, called a Coxeter diagram. The set of vertices of $\Gamma$ is $S$. Two vertices $s, t \in S$ are connected by an edge labeled by $m_{s, t}$ if $m_{s, t} \neq \infty$.

If $a, b$ are two letters and $m$ is an integer $\geq 2$, we set $\langle a, b\rangle^{m}=(a b)^{m / 2}$ if $m$ is even, and $\langle a, b\rangle^{m}=(a b)^{(m-1) / 2} a$ if $m$ is odd. In other words, $\langle a, b\rangle^{m}$ denotes the word $a b a \cdots$ of length $m$. The Artin group of $\Gamma$ is the group $A=A(\Gamma)$ defined by the presentation

$$
\left.A=\langle S|\langle s, t\rangle^{m_{s, t}}=\langle t, s\rangle^{m_{s, t}} \text { for all } s, t \in S, s \neq t \text { and } m_{s, t} \neq \infty\right\rangle .
$$

The Coxeter group of $\Gamma$, denoted by $W=W(\Gamma)$, is the quotient of $A$ by the relations $s^{2}=1, s \in S$.

Example. Let $\mathrm{V} \Gamma_{n}$ be the Coxeter diagram defined as follows. The set of vertices of $\mathrm{V} \Gamma_{n}$ is $\mathcal{S}$. If $i, j, k, l \in\{1, \ldots, n\}$ are distinct, then $\delta_{i, j}$ and $\delta_{k, l}$ are connected by an edge labeled by 2 . If $i, j, k \in\{1, \ldots, n\}$ are distinct, then $\delta_{i, j}$ and $\delta_{j, k}$ are connected by an edge labeled by 3 . There is no other edge in $\mathrm{V} \Gamma_{n}$. Then, by Proposition 2.1, $\mathrm{KB}_{n}$ is isomorphic to $A\left(\mathrm{~V} \Gamma_{n}\right)$. 
Let $\Gamma$ be a Coxeter diagram. For $X \subset S$, we denote by $\Gamma_{X}$ the subdiagram of $\Gamma$ spanned by $X$, by $A_{X}$ the subgroup of $A=A(\Gamma)$ generated by $X$, and by $W_{X}$ the subgroup of $W=W(\Gamma)$ generated by $X$. By [van der Lek 1983], $A_{X}$ is the Artin group of $\Gamma_{X}$, and, by [Bourbaki 1968], $W_{X}$ is the Coxeter group of $\Gamma_{X}$.

For $\mathcal{X} \subset \mathcal{S}$, we denote by $\mathrm{KB}_{n}(\mathcal{X})$ the subgroup of $\mathrm{KB}_{n}$ generated by $\mathcal{X}$. By the above, $\mathrm{KB}_{n}(\mathcal{X})$ has a presentation with generating set $\mathcal{X}$ and relations

- $s t=t s$ if $s$ and $t$ are connected in $\mathrm{V} \Gamma_{n}$ by an edge labeled by 2 ,

- $s t s=t s t$ if $s$ and $t$ are connected in $\mathrm{V} \Gamma_{n}$ by an edge labeled by 3 .

Definition. We say that a subset $\mathcal{X}$ of $\mathcal{S}$ is full if any two distinct elements $s, t$ of $\mathcal{X}$ are connected by an edge of $\mathrm{V} \Gamma_{n}$. (Recall that the aim of Step 2 is to give a solution to the word problem for $\mathrm{KB}_{n}(\mathcal{X})$ when $\mathcal{X}$ is full.)

We denote by $F_{n}=F\left(x_{1}, \ldots, x_{n}\right)$ the free group of rank $n$ freely generated by $x_{1}, \ldots, x_{n}$. For $i, j \in\{1, \ldots, n\}, i \neq j$, we define $\varphi_{i, j} \in \operatorname{Aut}\left(F_{n}\right)$ by

$$
\varphi_{i, j}\left(x_{i}\right)=x_{i} x_{j} x_{i}^{-1}, \quad \varphi_{i, j}\left(x_{j}\right)=x_{i} \quad \text { and } \quad \varphi_{i, j}\left(x_{k}\right)=x_{k} \text { for } k \notin\{i, j\} .
$$

It is easily seen from the presentation in Proposition 2.1 that the map $\mathcal{S} \rightarrow \operatorname{Aut}\left(F_{n}\right)$, $\delta_{i, j} \mapsto \varphi_{i, j}$, induces a representation $\varphi: \mathrm{KB}_{n} \rightarrow \operatorname{Aut}\left(F_{n}\right)$. For $\mathcal{X} \subset \mathcal{S}$, we denote by $\varphi_{\mathcal{X}}: \mathrm{KB}_{n}(\mathcal{X}) \rightarrow \operatorname{Aut}\left(F_{n}\right)$ the restriction of $\varphi$ to $\mathrm{KB}_{n}(\mathcal{X})$. The following will be proved in Section 3;

Proposition 2.3. If $\mathcal{X}$ is a full subset of $\mathcal{S}$, then $\varphi_{\mathcal{X}}: \mathrm{KB}_{n}(\mathcal{X}) \rightarrow \operatorname{Aut}\left(F_{n}\right)$ is faithful.

Notation. From now on, if $u$ is a word over $\mathcal{S}^{ \pm 1}$, then $\bar{u}$ will denote the element of $\mathrm{KB}_{n}$ represented by $u$.

Algorithm B. Let $\mathcal{X}$ be a full subset of $\mathcal{S}$ and let $u=s_{1}^{\varepsilon_{1}} \cdots s_{l}^{\varepsilon_{l}}$ be a word over $\mathcal{X}^{ \pm 1}$. We have $\varphi_{\mathcal{X}}(\bar{u})=\varphi_{\mathcal{X}}\left(s_{1}\right)^{\varepsilon_{1}} \cdots \varphi_{\mathcal{X}}\left(s_{l}\right)^{\varepsilon_{l}}$. If $\varphi(\bar{u})=\mathrm{Id}$, then $\bar{u}=1$. Otherwise, $\bar{u} \neq 1$.

Step 3. Let $G$ be a group, and let $H$ be a subgroup of $G$. A solution to the membership problem for $H$ in $G$ is an algorithm which, given $g \in G$, decides whether $g$ belongs to $H$ or not. In the present step we will assume that $\mathrm{KB}_{n}(\mathcal{X})$ has a solution to the word problem, and, from this solution, we will give a solution to the membership problem for $\operatorname{KB}_{n}(\mathcal{Y})$ in $\operatorname{KB}_{n}(\mathcal{X})$ for $\mathcal{Y} \subset \mathcal{X}$. Furthermore, if the tested element belongs to $\mathrm{KB}_{n}(\mathcal{Y})$, then this algorithm will determine a word over $\mathcal{Y}^{ \pm 1}$ which represents this element.

Let $u$ be a word over $\mathcal{S}$. (Remark: here the alphabet is $\mathcal{S}$, and not $\mathcal{S}^{ \pm 1}$.)

- Suppose that $u$ is written in the form $u_{1} s s u_{2}$, where $u_{1}, u_{2}$ are words over $\mathcal{S}$ and $s$ is an element of $\mathcal{S}$. Then we say that $u^{\prime}=u_{1} u_{2}$ is obtained from $u$ by an $M$-operation of type $\mathrm{I}$. 
- Suppose that $u$ is written in the form $u_{1}$ st $u_{2}$, where $u_{1}, u_{2}$ are words over $\mathcal{S}$ and $s, t$ are two elements of $\mathcal{S}$ connected by an edge labeled by 2 . Then we say that $u^{\prime}=u_{1} t s u_{2}$ is obtained from $u$ by an $M$-operation of type $\mathrm{II}^{(2)}$.

- Suppose that $u$ is written in the form $u_{1} s t s u_{2}$, where $u_{1}, u_{2}$ are words over $\mathcal{S}$ and $s, t$ are two elements of $\mathcal{S}$ connected by an edge labeled by 3 . Then we say that $u^{\prime}=u_{1}$ tst $u_{2}$ is obtained from $u$ by an $M$-operation of type $\mathrm{II}^{(3)}$.

Let $\mathcal{Y}$ be a subset of $\mathcal{S}$.

- Suppose that $u$ is written in the form $t u^{\prime}$, where $u^{\prime}$ is a word over $\mathcal{S}$ and $t$ is an element of $\mathcal{Y}$. Then we say that $u^{\prime}$ is obtained from $u$ by an $M$-operation of type $\mathrm{III} y$.

We say that $u$ is $M$-reduced (resp. My-reduced) if its length cannot be shortened by $M$-operations of type I, II ${ }^{(2)}$, II ${ }^{(3)}$ (resp. of type I, $\mathrm{II}^{(2)}, \mathrm{II}^{(3)}, \mathrm{III}_{\mathcal{Y}}$ ). An $M$-reduction (resp. $M_{\mathcal{Y}}$-reduction) of $u$ is an $M$-reduced word (resp. $M_{\mathcal{Y}}$-reduced word) obtained from $u$ by $M$-operations (resp. $M \mathcal{y}$-operations). We can easily enumerate all the words obtained from $u$ by $M$-operations (resp. $M_{\mathcal{y}}$-operations), hence we can effectively determine an $M$-reduction and/or an $M_{\mathcal{Y}}$-reduction of $u$.

Let $\mathcal{Y}$ be a subset of $\mathcal{S}$. From a word $u=s_{1}^{\varepsilon_{1}} \cdots s_{l}^{\varepsilon_{l}}$ over $\mathcal{S}^{ \pm 1}$, we construct a word $\pi_{\mathcal{Y}}(u)$ over $\mathcal{Y}^{ \pm 1}$ as follows:

- For $i \in\{0,1, \ldots, l\}$ we set $u_{i}^{+}=s_{1} \cdots s_{i}$ (as ever, $u_{0}^{+}$is the identity).

- For $i \in\{0,1, \ldots, l\}$ we calculate an $M \mathcal{Y}_{\text {-reduction }} v_{i}^{+}$of $u_{i}^{+}$.

- For a word $v=t_{1} \cdots t_{k}$ over $\mathcal{S}$, we let op $(v)=t_{k} \cdots t_{1}$. Let $i \in\{1, \ldots, l\}$. If $\varepsilon_{i}=1$, we set $w_{i}^{+}=v_{i-1}^{+} \cdot s_{i} \cdot \operatorname{op}\left(v_{i-1}^{+}\right)$. If $\varepsilon_{i}=-1$, we set $w_{i}^{+}=v_{i}^{+} \cdot s_{i} \cdot \operatorname{op}\left(v_{i}^{+}\right)$.

- For all $i \in\{1, \ldots, l\}$ we calculate an $M$-reduction $r_{i}$ of $w_{i}^{+}$.

- If $r_{i}$ is of length 1 and $r_{i} \in \mathcal{Y}$, we set $T_{i}=r_{i}^{\varepsilon_{i}}$. Otherwise we set $T_{i}=1$.

- We set $\pi_{Y}(u)=T_{1} T_{2} \cdots T_{l}$.

The proof of the following is given in Section 4.

Proposition 2.4. Let $\mathcal{Y}$ be a subset of $\mathcal{S}$. Let $u$, $v$ be two words over $\mathcal{S}^{ \pm 1}$. If $\bar{u}=\bar{v}$, then $\overline{\pi_{\mathcal{Y}}(u)}=\overline{\pi_{\mathcal{Y}}(v)}$. Moreover, we have $\bar{u} \in \mathrm{KB}_{n}(\mathcal{Y})$ if and only if $\bar{u}=\overline{\pi_{\mathcal{Y}}(u)}$.

Algorithm $\mathbf{C}$. Take two subsets $\mathcal{X}$ and $\mathcal{Y}$ of $\mathcal{S}$ such that $\mathcal{Y} \subset \mathcal{X}$, and assume given a solution to the word problem for $\operatorname{KB}_{n}(\mathcal{X})$. Let $u$ be a word over $\mathcal{X}^{ \pm 1}$. We calculate $v=\pi_{\mathcal{Y}}(u)$. If $\overline{u v^{-1}} \neq 1$, then $\bar{u} \notin \mathrm{KB}_{n}(\mathcal{Y})$. If $\overline{u v^{-1}}=1$, then $\bar{u} \in \mathrm{KB}_{n}(\mathcal{Y})$ and $v$ is a word over $\mathcal{Y}^{ \pm 1}$ which represents the same element as $u$.

We can use Algorithm $C$ to show that the representation $\varphi: \mathrm{KB}_{n} \rightarrow \operatorname{Aut}\left(F_{n}\right)$ of Step 2 is not faithful. Indeed, let $\alpha=\delta_{1,3} \delta_{3,2} \delta_{3,1}$ and $\beta=\delta_{2,3} \delta_{1,3} \delta_{3,2}$. A direct calculation shows that $\varphi(\alpha)=\varphi(\beta)$. Now, set $\mathcal{X}=\mathcal{S}$ and $\mathcal{Y}=\left\{\delta_{1,3}, \delta_{3,2}, \delta_{3,1}\right\}$. We have $\pi_{\mathcal{Y}}\left(\delta_{1,3} \delta_{3,2} \delta_{3,1}\right)=\delta_{1,3} \delta_{3,2} \delta_{3,1}$, hence $\alpha \in \mathrm{KB}_{n}(\mathcal{Y})$, and we have $\pi_{\mathcal{Y}}\left(\delta_{2,3} \delta_{1,3} \delta_{3,2}\right)=1$ and $\beta \neq 1$, hence $\beta \notin \mathrm{KB}_{n}(\mathcal{Y})$. Thus $\alpha \neq \beta$. 
Step 4. Now, we assume that $\mathcal{X}$ is a nonfull subset of $\mathcal{S}$, and that we have a solution to the word problem for $\operatorname{KB}_{n}(\mathcal{Y})$ for any proper subset $\mathcal{Y}$ of $\mathcal{X}$ (induction hypothesis). We can and do choose two proper subsets $\mathcal{X}_{1}, \mathcal{X}_{2} \subset \mathcal{X}$ satisfying the following properties:

(a) $\mathcal{X}=\mathcal{X}_{1} \cup \mathcal{X}_{2}$

(b) Let $\mathcal{X}_{0}=\mathcal{X}_{1} \cap \mathcal{X}_{2}$. There is no edge in $\mathrm{V} \Gamma_{n}$ connecting an element of $\mathcal{X}_{1} \backslash \mathcal{X}_{0}$ to an element of $\mathcal{X}_{2} \backslash \mathcal{X}_{0}$.

It is easily seen from the presentations of the $\mathrm{KB}_{n}\left(\mathcal{X}_{i}\right)$ given in Step 2 that we have the amalgamated product

$$
\mathrm{KB}_{n}(\mathcal{X})=\mathrm{KB}_{n}\left(\mathcal{X}_{1}\right) * \mathrm{~KB}_{n}\left(\mathcal{X}_{0}\right) \mathrm{KB}_{n}\left(\mathcal{X}_{2}\right) .
$$

Our last algorithm is based on the following result. This is well known and can be found for instance in [Serre 1977, Chapitre 5.2].

Proposition 2.5. Let $A_{1} *_{B} A_{2}$ be an amalgamated product of groups. Let $g_{1}, \ldots, g_{l}$ be a sequence of elements of $A_{1} \sqcup A_{2}$ different from 1 and satisfying the following condition:

if $g_{i} \in A_{1}$ (resp. $g_{i} \in A_{2}$ ) then $g_{i+1} \in A_{2} \backslash B$ (resp. $\left.g_{i+1} \in A_{1} \backslash B\right)$ for all $i \in\{1, \ldots, l-1\}$.

Then $g_{1} g_{2} \cdots g_{l}$ is different from 1 in $A_{1} *_{B} A_{2}$.

Algorithm D. Let $u$ be a word over $\mathcal{X}^{ \pm 1}$. We write $u$ in the form $u_{1} u_{2} \cdots u_{l}$, where

- $u_{i}$ is either a word over $\mathcal{X}_{1}^{ \pm 1}$ or a word over $\mathcal{X}_{2}^{ \pm 1}$,

- if $u_{i}$ is a word over $\mathcal{X}_{1}^{ \pm 1}$ (resp. over $\mathcal{X}_{2}^{ \pm 1}$ ), then $u_{i+1}$ is a word over $\mathcal{X}_{2}^{ \pm 1}$ (resp. over $\left.\mathcal{X}_{1}^{ \pm 1}\right)$.

We decide whether $\bar{u}$ is trivial by induction on $l$. Suppose that $l=1$ and $u=$ $u_{1} \in \mathrm{KB}_{n}\left(\mathcal{X}_{j}\right)(j \in\{1,2\})$. Then we apply the solution to the word problem for $\mathrm{KB}_{n}\left(\mathcal{X}_{j}\right)$ to decide whether $\bar{u}$ is trivial or not. Suppose that $l \geq 2$. For all $i$ we set $v_{i}=\pi_{\mathcal{X}_{0}}\left(u_{i}\right)$. If $\overline{u_{i} v_{i}^{-1}} \neq 1$ for all $i$, then $\bar{u} \neq 1$. Suppose there exists an integer $i \in\{1, \ldots, l\}$ such that $\overline{u_{i} v_{i}^{-1}}=1$. Let $u_{i}^{\prime}=v_{1} u_{2}$ if $i=1, u_{i}^{\prime}=u_{l-1} v_{l}$ if $i=l$, and $u_{i}^{\prime}=u_{i-1} v_{i} u_{i+1}$ if $2 \leq i \leq l-1$. Set $v=u_{1} \cdots u_{i-2} u_{i}^{\prime} u_{i+2} \cdots u_{l}$. Then $\bar{u}=\bar{v}$ and, by induction, we can decide whether $v$ represents 1 or not.

Example. In order to illustrate our solution to the word problem for $\mathrm{KB}_{n}$, we turn now to give a more detailed and efficient version of the algorithm for the group $\mathrm{KB}_{4}$. We start with the following observation:

Remark. For $\mathcal{X} \subset \mathcal{S}$, we denote by $\mathrm{V} \Gamma_{n}(\mathcal{X})$ the full subgraph of $\mathrm{V} \Gamma_{n}$ spanned by $\mathcal{X}$. Let $\mathcal{X}, \mathcal{Y}$ be two subsets of $\mathcal{S}$. Note that an injective morphism of Coxeter graphs $\mathrm{V} \Gamma_{n}(\mathcal{Y}) \hookrightarrow \mathrm{V} \Gamma_{n}(\mathcal{X})$ induces an injective homomorphism $\mathrm{KB}_{n}(\mathcal{Y}) \hookrightarrow \mathrm{KB}_{n}(\mathcal{X})$. So, 


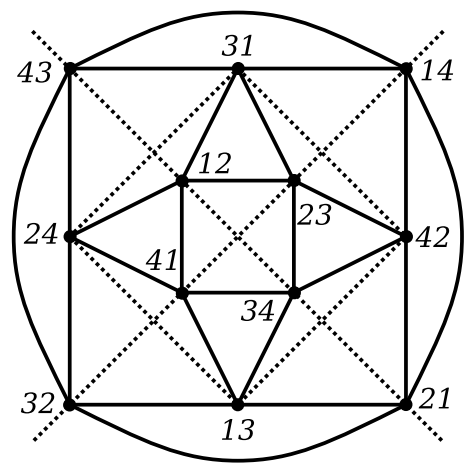

Figure 4. Coxeter graph $\mathrm{V} \Gamma_{4}$.

if we had a solution to the word problem for $\mathrm{KB}_{n}(\mathcal{X})$, then such a morphism would determine a solution to the word problem for $\mathrm{KB}_{n}(\mathcal{Y})$.

The Coxeter graph $\mathrm{V}_{4}$ is depicted in Figure 4. Our convention in this figure is that a full edge is labeled by 3 and a dotted edge is labeled by 2 . Note that there are two edges that go through "infinity", one connecting $\delta_{2,1}$ to $\delta_{4,3}$, and one connecting $\delta_{1,4}$ to $\delta_{3,2}$.

Consider the following subsets of $\mathcal{S}$ :

$$
\begin{aligned}
& \mathcal{X}^{(1)}=\left\{\delta_{1,2}, \delta_{2,3}, \delta_{3,4}, \delta_{4,1}, \delta_{3,1}\right\}, \mathcal{X}_{1}^{(1)}=\left\{\delta_{1,2}, \delta_{2,3}, \delta_{3,4}, \delta_{4,1}\right\}, \mathcal{X}_{2}^{(1)}=\left\{\delta_{1,2}, \delta_{2,3}, \delta_{3,1}\right\} . \\
& \mathcal{X}^{(2)}=\mathcal{X}^{(1)} \cup\left\{\delta_{4,2}\right\}, \mathcal{X}_{1}^{(2)}=\mathcal{X}^{(1)}, \mathcal{X}_{2}^{(2)}=\left\{\delta_{4,2}, \delta_{3,4}, \delta_{2,3}, \delta_{3,1}\right\} . \\
& \mathcal{X}^{(3)}=\mathcal{X}^{(2)} \cup\left\{\delta_{1,3}\right\}, \mathcal{X}_{1}^{(3)}=\mathcal{X}^{(2)}, \mathcal{X}_{2}^{(3)}=\left\{\delta_{1,3}, \delta_{4,1}, \delta_{3,4}, \delta_{4,2}\right\} . \\
& \mathcal{X}^{(4)}=\mathcal{X}^{(3)} \cup\left\{\delta_{2,4}\right\}, \mathcal{X}_{1}^{(4)}=\mathcal{X}^{(3)}, \mathcal{X}_{2}^{(4)}=\left\{\delta_{2,4}, \delta_{1,3}, \delta_{4,1}, \delta_{1,2}, \delta_{3,1}\right\} . \\
& \mathcal{X}^{(5)}=\mathcal{X}^{(4)} \cup\left\{\delta_{1,4}\right\}, \mathcal{X}_{1}^{(5)}=\mathcal{X}^{(4)}, \mathcal{X}_{2}^{(5)}=\left\{\delta_{1,4}, \delta_{4,2}, \delta_{2,3}, \delta_{3,1}\right\} . \\
& \mathcal{X}^{(6)}=\mathcal{X}^{(5)} \cup\left\{\delta_{2,1}\right\}, \mathcal{X}_{1}^{(6)}=\mathcal{X}^{(5)}, \mathcal{X}_{2}^{(6)}=\left\{\delta_{2,1}, \delta_{1,3}, \delta_{3,4}, \delta_{4,2}, \delta_{1,4}\right\} . \\
& \mathcal{X}^{(7)}=\mathcal{X}^{(6)} \cup\left\{\delta_{3,2}\right\}, \mathcal{X}_{1}^{(7)}=\mathcal{X}^{(6)}, \mathcal{X}_{2}^{(7)}=\left\{\delta_{3,2}, \delta_{2,4}, \delta_{4,1}, \delta_{1,3}, \delta_{2,1}, \delta_{1,4}\right\} . \\
& \mathcal{X}^{(8)}=\mathcal{X}^{(7)} \cup\left\{\delta_{4,3}\right\}=\mathcal{S}, \mathcal{X}_{1}^{(8)}=\mathcal{X}^{(7)}, \mathcal{X}_{2}^{(8)}=\left\{\delta_{4,3}, \delta_{3,2}, \delta_{2,4}, \delta_{1,2}, \delta_{3,1}, \delta_{1,4}, \delta_{2,1}\right\} .
\end{aligned}
$$

Let $k \in\{1, \ldots, 8\}$. Note that $\mathcal{X}^{(k)}=\mathcal{X}_{1}^{(k)} \cup \mathcal{X}_{2}^{(k)}$. The Coxeter graph $\mathrm{V} \Gamma_{4}\left(\mathcal{X}^{(k)}\right)$ is depicted in Figure 5. In this figure the elements of $\mathcal{X}_{1}^{(k)}$ are represented by punctures, while the elements of $\mathcal{X}_{2}^{(k)}$ are represented by small circles.

We solve the word problem for $\mathrm{KB}_{4}\left(\mathcal{X}^{(k)}\right)$ successively for $k=1,2, \ldots, 8$, thanks to the following observations. Since $\mathcal{X}^{(8)}=\mathcal{S}$, this will provide a solution to the word problem for $\mathrm{KB}_{4}$.

(1) Let $k \in\{1, \ldots, 8\}$. Set $\mathcal{X}_{0}^{(k)}=\mathcal{X}_{1}^{(k)} \cap \mathcal{X}_{2}^{(k)}$. Observe that there is no edge in $\mathrm{V} \Gamma_{4}$ connecting an element of $\mathcal{X}_{1}^{(k)} \backslash \mathcal{X}_{0}^{(k)}$ to an element of $\mathcal{X}_{2}^{(k)} \backslash \mathcal{X}_{0}^{(k)}$. 

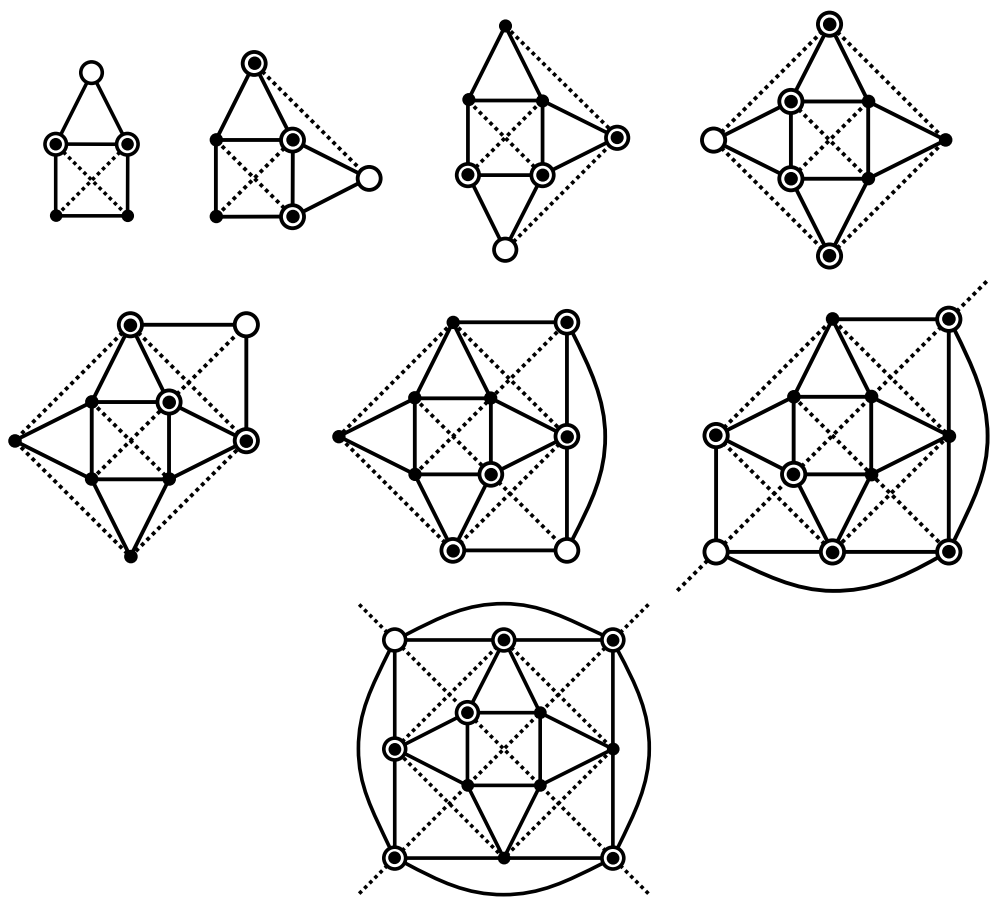

Figure 5. Coxeter graphs $\mathrm{V} \Gamma_{4}\left(\mathcal{X}^{(k)}\right)$ for $k=1, \ldots, 8$ from left to right.

Hence, we can solve, using Algorithm D, the word problem for $\mathrm{KB}_{4}\left(\mathcal{X}^{(k)}\right)$ from solutions to the word problem for $\mathrm{KB}_{4}\left(\mathcal{X}_{1}^{(k)}\right)$ and for $\mathrm{KB}_{4}\left(\mathcal{X}_{2}^{(k)}\right)$.

(2) The subsets $\mathcal{X}_{1}^{(1)}$ and $\mathcal{X}_{2}^{(1)}$ are full, hence we can solve the word problem for $\mathrm{KB}_{4}\left(\mathcal{X}_{1}^{(1)}\right)$ and for $\mathrm{KB}_{4}\left(\mathcal{X}_{2}^{(1)}\right)$ with Algorithm B.

(3) Let $k \geq 2$. On the one hand, we have $\mathcal{X}_{1}^{(k)}=\mathcal{X}^{(k-1)}$. On the other hand, it is easily seen that there is an injective morphism $\mathrm{V} \Gamma_{4}\left(\mathcal{X}_{2}^{(k)}\right) \hookrightarrow \mathrm{V} \Gamma_{4}\left(\mathcal{X}^{(k-1)}\right)$. Hence, by the remark given at the beginning of the subsection, we can solve the word problem for $\mathrm{KB}_{4}\left(\mathcal{X}_{1}^{(k)}\right)$ and for $\mathrm{KB}_{4}\left(\mathcal{X}_{2}^{(k)}\right)$ from a solution to the word problem for $\mathrm{KB}_{4}\left(\mathcal{X}^{(k-1)}\right)$.

\section{Proof of Proposition 2.3}

Recall that $F_{n}=F\left(x_{1}, \ldots, x_{n}\right)$ denotes the free group of rank $n$ freely generated by $x_{1}, \ldots, x_{n}$, and that we have a representation $\varphi: \mathrm{KB}_{n} \rightarrow \operatorname{Aut}\left(F_{n}\right)$ which sends $\delta_{i, j}$ to $\varphi_{i, j}$, where

$$
\varphi_{i, j}\left(x_{i}\right)=x_{i} x_{j} x_{i}^{-1}, \quad \varphi_{i, j}\left(x_{j}\right)=x_{i} \quad \text { and } \quad \varphi_{i, j}\left(x_{k}\right)=x_{k} \text { for } k \notin\{i, j\} .
$$

For $\mathcal{X} \subset \mathcal{S}$, we denote by $\varphi_{\mathcal{X}}: \mathrm{KB}_{n}(\mathcal{X}) \rightarrow \operatorname{Aut}\left(F_{n}\right)$ the restriction of $\varphi$ to $\mathrm{KB}_{n}(\mathcal{X})$. In this section we prove that $\varphi_{\mathcal{X}}$ is faithful if $\mathcal{X}$ is a full subset of $\mathcal{S}$. 
Consider the groups

$$
\begin{aligned}
B_{n} & =\left\langle\sigma_{1}, \ldots, \sigma_{n-1} \mid \begin{array}{cl}
\sigma_{i} \sigma_{j} \sigma_{i}=\sigma_{j} \sigma_{i} \sigma_{j} & \text { if }|i-j|=1 \\
\sigma_{i} \sigma_{j}=\sigma_{j} \sigma_{i} & \text { if }|i-j| \geq 2
\end{array}\right\rangle, \\
\tilde{B}_{n} & =\left\langle\begin{array}{cc}
\sigma_{1}, \ldots, \sigma_{n}\left|\begin{array}{cc}
\sigma_{i} \sigma_{j} \sigma_{i}=\sigma_{j} \sigma_{i} \sigma_{j} & \text { if } i \equiv j \pm 1 \bmod n \\
\sigma_{i} \sigma_{j}=\sigma_{j} \sigma_{i} & \text { if } i \neq j \text { and } i \neq \equiv j \pm 1 \bmod n
\end{array}\right\rangle, \quad n \geq 3 .
\end{array}\right.
\end{aligned}
$$

The group $B_{n}$ is the classical braid group, and $\tilde{B}_{n}$ is the affine braid group.

We define representations $\psi_{n}: B_{n} \rightarrow \operatorname{Aut}\left(F_{n}\right)$ and $\tilde{\psi}_{n}: \tilde{B}_{n} \rightarrow \operatorname{Aut}\left(F_{n}\right)$ in the same way as $\varphi$, as follows:

$$
\begin{gathered}
\psi_{n}\left(\sigma_{i}\right)\left(x_{i}\right)=x_{i} x_{i+1} x_{i}^{-1}, \quad \psi_{n}\left(\sigma_{i}\right)\left(x_{i+1}\right)=x_{i}, \quad \psi_{n}\left(\sigma_{i}\right)\left(x_{k}\right)=x_{k} \text { if } k \notin\{i, i+1\}, \\
\tilde{\psi}_{n}\left(\sigma_{i}\right)=\psi_{n}\left(\sigma_{i}\right) \text { for } i<n, \\
\tilde{\psi}_{n}\left(\sigma_{n}\right)\left(x_{n}\right)=x_{n} x_{1} x_{n}^{-1}, \quad \tilde{\psi}_{n}\left(\sigma_{n}\right)\left(x_{1}\right)=x_{n}, \quad \tilde{\psi}_{n}\left(\sigma_{n}\right)\left(x_{k}\right)=x_{k} \text { if } k \notin\{1, n\},
\end{gathered}
$$

The key of the proof of Proposition 2.3 is the following:

Theorem 3.1 [Artin 1947; Bellingeri and Bodin 2016]. The representations $\psi_{n}$ : $B_{n} \rightarrow \operatorname{Aut}\left(F_{n}\right)$ and $\tilde{\psi}_{n}: \tilde{B}_{n} \rightarrow \operatorname{Aut}\left(F_{n}\right)$ are faithful.

The support of a generator $\delta_{i, j}$ is defined to be $\operatorname{supp}\left(\delta_{i, j}\right)=\{i, j\}$. The support of a subset $\mathcal{X}$ of $\mathcal{S}$ is $\operatorname{supp}(\mathcal{X})=\bigcup_{s \in \mathcal{X}} \operatorname{supp}(s)$. We say that two subsets $\mathcal{X}_{1}$ and $\mathcal{X}_{2}$ of $\mathcal{S}$ are perpendicular ${ }^{1}$ if $\operatorname{supp}\left(\mathcal{X}_{1}\right) \cap \operatorname{supp}\left(\mathcal{X}_{2}\right)=\varnothing$. Note that this condition implies that $\mathcal{X}_{1} \cap \mathcal{X}_{2}=\varnothing$. More generally, we say that a family $\mathcal{X}_{1}, \ldots, \mathcal{X}_{l}$ of subsets of $\mathcal{S}$ is perpendicular if $\operatorname{supp}\left(\mathcal{X}_{i}\right) \cap \operatorname{supp}\left(\mathcal{X}_{j}\right)=\varnothing$ for all $i \neq j$. In that case we write $\mathcal{X}_{1} \cup \cdots \cup \mathcal{X}_{l}=\mathcal{X}_{1} \boxplus \cdots \boxplus \mathcal{X}_{l}$. We say that a subset $\mathcal{X}$ of $\mathcal{S}$ is indecomposable if it is not the union of two perpendicular nonempty subsets. The next observations will be of importance in what follows.

Remarks. Let $\mathcal{X}_{1}$ and $\mathcal{X}_{2}$ be two perpendicular subsets of $\mathcal{S}$, and let $\mathcal{X}=\mathcal{X}_{1} \boxplus \mathcal{X}_{2}$.

(1) $\mathcal{X}$ is a full subset if and only if $\mathcal{X}_{1}$ and $\mathcal{X}_{2}$ are both full subsets.

(2) $\mathrm{KB}_{n}(\mathcal{X})=\mathrm{KB}_{n}\left(\mathcal{X}_{1}\right) \times \mathrm{KB}_{n}\left(\mathcal{X}_{2}\right)$.

Indeed, if $\delta_{i, j} \in \mathcal{X}_{1}$ and $\delta_{k, l} \in \mathcal{X}_{2}$, then $i, j, k, l$ are distinct, and therefore $\delta_{i, j}$ and $\delta_{k, l}$ are connected by an edge labeled by 2 , and $\delta_{i, j} \delta_{k, l}=\delta_{k, l} \delta_{i, j}$.

Lemma 3.2. Let $\mathcal{X}_{1}$ and $\mathcal{X}_{2}$ be two perpendicular subsets of $\mathcal{S}$, and let $\mathcal{X}=\mathcal{X}_{1} \boxplus \mathcal{X}_{2}$. Then $\varphi_{\mathcal{X}}: \mathrm{KB}_{n}(\mathcal{X}) \rightarrow \operatorname{Aut}\left(F_{n}\right)$ is faithful if and only if $\varphi_{\mathcal{X}_{1}}: \mathrm{KB}_{n}\left(\mathcal{X}_{1}\right) \rightarrow \operatorname{Aut}\left(F_{n}\right)$ and $\varphi_{\mathcal{X}_{2}}: \mathrm{KB}_{n}\left(\mathcal{X}_{2}\right) \rightarrow \operatorname{Aut}\left(F_{n}\right)$ are both faithful.

${ }^{1}$ This terminology is derived from the theory of Coxeter groups. 
Proof. For $X \subset\left\{x_{1}, \ldots, x_{n}\right\}$, we denote by $F(X)$ the subgroup of $F_{n}$ generated by $X$. There is a natural embedding $\iota_{X}: \operatorname{Aut}(F(X)) \hookrightarrow \operatorname{Aut}\left(F_{n}\right)$ defined by

$$
\iota_{X}(\alpha)\left(x_{i}\right)= \begin{cases}\alpha\left(x_{i}\right) & \text { if } x_{i} \in X \\ x_{i} & \text { otherwise }\end{cases}
$$

Moreover, if $X_{1}$ and $X_{2}$ are disjoint subsets of $\left\{x_{1}, \ldots, x_{n}\right\}$, then the homomorphism

$$
\begin{aligned}
\left(\iota_{X_{1}} \times \iota_{X_{2}}\right): \operatorname{Aut}\left(F\left(X_{1}\right)\right) \times \operatorname{Aut}\left(F\left(X_{2}\right)\right) & \rightarrow \operatorname{Aut}\left(F_{n}\right), \\
\left(\alpha_{1}, \alpha_{2}\right) & \mapsto \iota_{X_{1}}\left(\alpha_{1}\right) \iota_{X_{2}}\left(\alpha_{2}\right),
\end{aligned}
$$

is well-defined and injective. From now on, we will assume $\operatorname{Aut}(F(X))$ to be embedded in $\operatorname{Aut}\left(F_{n}\right)$ via $\iota_{X}$ for all $X \subset\left\{x_{1}, \ldots, x_{n}\right\}$.

By an abuse of notation, for $\mathcal{X} \subset \mathcal{S}$ we will also denote by $\operatorname{supp}(\mathcal{X})$ the set $\left\{x_{i} \mid i \in \operatorname{supp}(\mathcal{X})\right\}$. Set $X_{1}=\operatorname{supp}\left(\mathcal{X}_{1}\right)$ and $X_{2}=\operatorname{supp}\left(\mathcal{X}_{2}\right)$. We have $\operatorname{Im}\left(\varphi_{\mathcal{X}_{i}}\right) \subset$ $\operatorname{Aut}\left(F\left(X_{i}\right)\right)$ for $i=1,2, X_{1} \cap X_{2}=\varnothing$, and $\mathrm{KB}_{n}(\mathcal{X})=\mathrm{KB}_{n}\left(\mathcal{X}_{1}\right) \times \mathrm{KB}_{n}\left(\mathcal{X}_{2}\right)$. Hence, Lemma 3.2 follows from the following claim, whose proof is left to the reader:

Let $f_{1}: G_{1} \rightarrow H_{1}$ and $f_{2}: G_{2} \rightarrow H_{2}$ be two group homomorphisms. Let $\left(f_{1} \times f_{2}\right)$ : $\left(G_{1} \times G_{2}\right) \rightarrow\left(H_{1} \times H_{2}\right)$ be the homomorphism defined by $\left(f_{1} \times f_{2}\right)\left(u_{1}, u_{2}\right)=$ $\left(f_{1}\left(u_{1}\right), f_{2}\left(u_{2}\right)\right)$. Then $\left(f_{1} \times f_{2}\right)$ is injective if and only if $f_{1}$ and $f_{2}$ are both injective.

For $2 \leq m \leq n$ we set

$$
\mathcal{Z}_{m}=\left\{\delta_{1,2}, \ldots, \delta_{m-1, m}\right\}, \quad \tilde{\mathcal{Z}}_{m}=\left\{\delta_{1,2}, \ldots, \delta_{m-1, m}, \delta_{m, 1}\right\} .
$$

Note that the map $\left\{\sigma_{1}, \ldots, \sigma_{m-1}\right\} \rightarrow \mathcal{Z}_{m}, \sigma_{i} \mapsto \delta_{i, i+1}$, induces an isomorphism $f_{m}: B_{m} \rightarrow \operatorname{KB}_{n}\left(\mathcal{Z}_{m}\right)$. This follows from the presentation of $\operatorname{KB}_{n}\left(\mathcal{Z}_{m}\right)$ given in Step 2 of Section 2. Similarly, for $m \geq 3$ the map $\left\{\sigma_{1}, \ldots, \sigma_{m}\right\} \rightarrow \tilde{\mathcal{Z}}_{m}, \sigma_{i} \mapsto \delta_{i, i+1}$ for $1 \leq i \leq m-1, \sigma_{m} \mapsto \delta_{m, 1}$, induces an isomorphism $\tilde{f}_{m}: \tilde{B}_{m} \rightarrow \operatorname{KB}_{n}\left(\tilde{\mathcal{Z}}_{m}\right)$.

Recall that the symmetric group $\mathfrak{S}_{n}$ acts on $\mathcal{S}$ by $g \delta_{i, j}=\delta_{g(i), g(j)}$, and that this action induces an action of $\mathfrak{S}_{n}$ on $\mathrm{KB}_{n}$. On the other hand, there is a natural embedding $\mathfrak{S}_{n} \hookrightarrow \operatorname{Aut}\left(F_{n}\right)$, where $g \in \mathfrak{S}_{n}$ sends $x_{i}$ to $x_{g(i)}$ for all $i \in\{1, \ldots, n\}$, and this embedding induces by conjugation an action of $\mathfrak{S}_{n}$ on $\operatorname{Aut}\left(F_{n}\right)$. It is easily seen that the homomorphism $\varphi: \mathrm{KB}_{n} \rightarrow \operatorname{Aut}\left(F_{n}\right)$ is equivariant under these actions of $\mathfrak{S}_{n}$.

Lemma 3.3. If $\mathcal{X}$ is a full and indecomposable nonempty subset of $\mathcal{S}$, then there exist $g \in \mathfrak{S}_{n}$ and $m \in\{2, \ldots, n\}$ such that either $\mathcal{X}=g \mathcal{Z}_{m}$, or $\mathcal{X}=g \tilde{\mathcal{Z}}_{m}$ and $m \geq 3$. Proof. An oriented graph $\Upsilon$ is the data of two sets, $V(\Upsilon)$, called the set of vertices, and $E(\Upsilon)$, called the set of arrows, together with two maps sou, $\operatorname{tar}: E(\Upsilon) \rightarrow V(\Upsilon)$. We associate an oriented graph $\Upsilon_{\mathcal{X}}$ to any subset $\mathcal{X}$ of $\mathcal{S}$ as follows. The set of vertices is $V\left(\Upsilon_{\mathcal{X}}\right)=\operatorname{supp}(\mathcal{X})$, the set of arrows is $E\left(\Upsilon_{\mathcal{X}}\right)=\mathcal{X}$, and, for $\delta_{i, j} \in \mathcal{X}$, we set $\operatorname{sou}\left(\delta_{i, j}\right)=i$ and $\operatorname{tar}\left(\delta_{i, j}\right)=j$. Assume that $\mathcal{X}$ is a full and indecomposable 

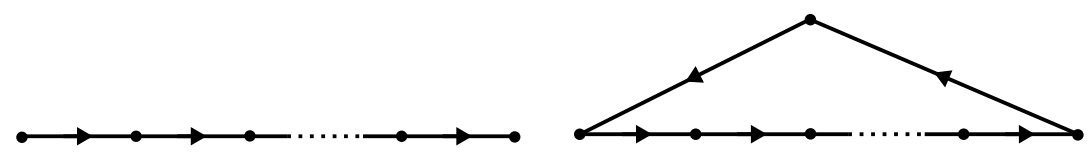

Figure 6. Oriented segment and oriented cycle.

nonempty subset of $\mathcal{S}$. Since $\mathcal{X}$ is indecomposable, $\Upsilon_{\mathcal{X}}$ must be connected. Since $\mathcal{X}$ is full, if $s, t \in \mathcal{X}$ are two different arrows of $\Upsilon_{\mathcal{X}}$ with a common vertex, then there exist $i, j, k \in\{1, \ldots, n\}$ distinct such that either $s=\delta_{j, i}$ and $t=\delta_{i, k}$, or $s=\delta_{i, j}$ and $t=\delta_{k, i}$. This implies that $\Upsilon_{\mathcal{X}}$ is either an oriented segment, or an oriented cycle with at least 3 vertices (see Figure 6). If $\Upsilon_{\mathcal{X}}$ is an oriented segment, then there exist $g \in \mathfrak{S}_{n}$ and $m \in\{2, \ldots, n\}$ such that $\mathcal{X}=g \mathcal{Z}_{m}$. If $\Upsilon_{\mathcal{X}}$ is an oriented cycle, then there exist $g \in \mathfrak{S}_{n}$ and $m \in\{3, \ldots, n\}$, such that $\mathcal{X}=g \tilde{\mathcal{Z}}_{m}$.

Proof of Proposition 2.3. Let $\mathcal{X}$ be a full nonempty subset of $\mathcal{S}$. Write $\mathcal{X}=$ $\mathcal{X}_{1} \boxplus \cdots \boxplus \mathcal{X}_{l}$, where $\mathcal{X}_{j}$ is an indecomposable nonempty subset. As observed above, each $\mathcal{X}_{j}$ is also a full subset. Moreover, by Lemma 3.2, in order to show that $\varphi_{\mathcal{X}}$ is faithful, it suffices to show that $\varphi_{\mathcal{X}}$ is faithful for all $j \in\{1, \ldots, l\}$. So, we can assume that $\mathcal{X}$ is a full and indecomposable nonempty subset of $\mathcal{S}$. By Lemma 3.3, there exist $g \in \mathfrak{S}_{n}$ and $m \in\{2, \ldots, n\}$ such that either $\mathcal{X}=g \mathcal{Z}_{m}$, or $\mathcal{X}=g \tilde{\mathcal{Z}}_{m}$ and $m \geq 3$. Since $\varphi$ is equivariant under the actions of $\mathfrak{S}_{n}$, upon conjugating by $g^{-1}$ we can assume that either $\mathcal{X}=\mathcal{Z}_{m}$ or $\mathcal{X}=\tilde{\mathcal{Z}}_{m}$. Set $Z_{m}=\left\{x_{1}, \ldots, x_{m}\right\}=$ $\operatorname{supp}\left(\mathcal{Z}_{m}\right)=\operatorname{supp}\left(\tilde{\mathcal{Z}}_{m}\right)$, and identify $F_{m}$ with $F\left(Z_{m}\right)$. Then $\varphi_{\mathcal{Z}_{m}}=\psi_{m} \circ f_{m}^{-1}$ and $\varphi_{\tilde{Z}_{m}}=\tilde{\psi}_{m} \circ \tilde{f}_{m}^{-1}$, hence $\varphi_{\mathcal{X}}$ is faithful by Theorem 3.1 .

\section{Proof of Proposition 2.4}

The proof of Proposition 2.4 is based on some general results on Coxeter groups and Artin groups. Recall that the definitions of Coxeter diagram, Artin group and Coxeter group are given at the beginning of Step 2 in Section 2. Recall also that, if $Y$ is a subset of the set $S$ of vertices of $\Gamma$, then $\Gamma_{Y}$ denotes the full subdiagram spanned by $Y, A_{Y}$ denotes the subgroup of $A=A(\Gamma)$ generated by $Y$, and $W_{Y}$ denotes the subgroup of $W=W(\Gamma)$ generated by $Y$.

Let $\Gamma$ be a Coxeter diagram, let $S$ be its set of vertices, let $A$ be the Artin group of $\Gamma$, and let $W$ be its Coxeter group. Since we have $s^{2}=1$ in $W$ for all $s \in S$, every element $g$ in $W$ can be represented by a word over $S$. Such a word is called an expression of $g$. The minimal length of an expression of $g$ is called the length of $g$ and is denoted by $\lg (g)$. An expression of $g$ of length $\lg (g)$ is a reduced expression of $g$. Let $Y$ be a subset of $S$, and let $g \in W$. We say that $g$ is $Y$-minimal if it is of minimal length among the elements of the coset $W_{Y} g$. The first ingredient in our proof of Proposition 2.4 is the following: 
Proposition 4.1 [Bourbaki 1968, Chapitre IV, Exercice 3]. Let $Y \subset S$ and let $g \in W$. There exists a unique $Y$-minimal element lying in the coset $W_{Y} g$. Moreover, the following conditions are equivalent:

(a) $g$ is $Y$-minimal.

(b) $\lg (s g)>\lg (g)$ for all $s \in Y$.

(c) $\lg (h g)=\lg (h)+\lg (g)$ for all $h \in W_{Y}$.

Remark. For $g \in W$ and $s \in S$, we always have either $\lg (s g)=\lg (g)+1$, or $\lg (s g)=\lg (g)-1$. This is a standard fact on Coxeter groups that can be found for instance in [Bourbaki 1968]. So, the inequality $\lg (s g)>\lg (g)$ means $\lg (s g)=$ $\lg (g)+1$ and the inequality $\lg (s g) \leq \lg (g)$ means $\lg (s g)=\lg (g)-1$.

Let $u$ be a word over $S$.

- Suppose that $u$ is written in the form $u_{1} s s u_{2}$, where $u_{1}, u_{2}$ are words over $S$ and $s$ is an element of $S$. Then we say that $u^{\prime}=u_{1} u_{2}$ is obtained from $u$ by an $M$-operation of type $\mathrm{I}$.

- Suppose that $u$ is written in the form $u=u_{1}\langle s, t\rangle^{m_{s, t}} u_{2}$, where $u_{1}, u_{2}$ are words over $S$ and $s, t$ are two elements of $S$ connected by an edge labeled by $m_{s, t}$. Then we say that $u^{\prime}=u_{1}\langle t, s\rangle^{m_{s, t}} u_{2}$ is obtained from $u$ by an $M$-operation of type II.

We say that a word $u$ is $M$-reduced if its length cannot be shortened by $M$-operations of types I or II. The second ingredient in our proof is the following.

Theorem 4.2 [Tits 1969]. Let $g \in W$.

(1) An expression $w$ of $g$ is a reduced expression if and only if $w$ is $M$-reduced.

(2) Any two reduced expressions $w$ and $w^{\prime}$ of $g$ are connected by a finite sequence of $M$-operations of type II.

Let $Y$ be a subset of $S$. The third ingredient is a set retraction $\rho_{Y}: A \rightarrow A_{Y}$ to the inclusion map $\iota_{Y}: A_{Y} \rightarrow A$, constructed in [Godelle and Paris 2012; Charney and Paris 2014]. This is defined as follows. Let $\alpha$ be an element of $A$.

- Choose a word $\hat{\alpha}=s_{1}{ }^{\varepsilon_{1}} \cdots s_{l}{ }^{\varepsilon_{l}}$ over $S^{ \pm 1}$ which represents $\alpha$.

- Let $i \in\{0,1, \ldots, l\}$. Set $g_{i}=s_{1} s_{2} \cdots s_{i} \in W$, and write $g_{i}$ in the form $g_{i}=h_{i} k_{i}$, where $h_{i} \in W_{Y}$ and $k_{i}$ is $Y$-minimal.

- Let $i \in\{1, \ldots, l\}$. If $\varepsilon_{i}=1$, set $z_{i}=k_{i-1} s_{i} k_{i-1}^{-1}$. If $\varepsilon_{i}=-1$, set $z_{i}=k_{i} s_{i} k_{i}^{-1}$.

- Let $i \in\{1, \ldots, l\}$. We set $T_{i}=z_{i}^{\varepsilon_{i}}$ if $z_{i} \in Y$. Otherwise we set $T_{i}=1$.

- Set $\hat{\rho}_{Y}(\alpha)=T_{1} T_{2} \cdots T_{l}$. 
Proposition 4.3 [Godelle and Paris 2012; Charney and Paris 2014]. Let $\alpha \in A$. The element $\rho_{Y}(\alpha) \in A_{Y}$ represented by the word $\hat{\rho}_{Y}(\alpha)$ defined above does not depend on the choice of the representative $\hat{\alpha}$ of $\alpha$. Furthermore, the map $\rho_{Y}: A \rightarrow A_{Y}$ is a set retraction to the inclusion map $\iota_{Y}: A_{Y} \hookrightarrow A$.

We turn now to apply these three ingredients to our group $\mathrm{KB}_{n}$ and prove Proposition 2.4. Let $\mathrm{KW}_{n}$ denote the quotient of $\mathrm{KB}_{n}$ by the relations $\delta_{i, j}^{2}=1$, $1 \leq i \neq j \leq n$. Note that $\mathrm{KW}_{n}$ is the Coxeter group of the Coxeter diagram $\mathrm{V} \Gamma_{n}$. For $\mathcal{Y} \subset \mathcal{X}$, we denote by $\mathrm{KW}_{n}(\mathcal{Y})$ the subgroup of $\mathrm{KW}_{n}$ generated by $\mathcal{Y}$.

\section{Lemma 4.4. Let $g \in \mathrm{KW}_{n}$.}

(1) An expression $w$ of $g$ is a reduced expression if and only if $w$ is $M$-reduced.

(2) Any two reduced expressions $w$ and $w^{\prime}$ of $g$ are connected by a finite sequence of $M$-operations of types $\mathrm{II}^{(2)}$ and $\mathrm{II}^{(3)}$.

(3) Let $\mathcal{Y}$ be a subset of $\mathcal{S}$, and let $w$ be a reduced expression of $g$. Then $g$ is $\mathcal{Y}$-minimal (in the sense given above) if and only if $w$ is $M_{\mathcal{Y}}$-reduced.

Proof. Parts (1) and (2) are Theorem 4.2 applied to $\mathrm{KW}_{n}$. So, we only need to prove (3).

Suppose that $g$ is not $\mathcal{Y}$-minimal. By Proposition 4.1, there exists $s \in \mathcal{Y}$ such that $\lg (s g) \leq \lg (g)$, that is, $\lg (s g)=\lg (g)-1$. Let $w^{\prime}$ be a reduced expression of $s g$. The word $s w^{\prime}$ is an expression of $g$ and $\lg \left(s w^{\prime}\right)=\lg (w)=\lg (g)$, hence $s w^{\prime}$ is a reduced expression of $g$. By Theorem 4.2, $w$ and $s w^{\prime}$ are connected by a finite sequence of $M$-operations of types $\mathrm{II}^{(2)}$ and $\mathrm{II}^{(3)}$. On the other hand, $w^{\prime}$ is obtained from $s w^{\prime}$ by an $M$-operation of type $\mathrm{III}_{\mathcal{Y}}$. So, $w^{\prime}$ is obtained from $w$ by

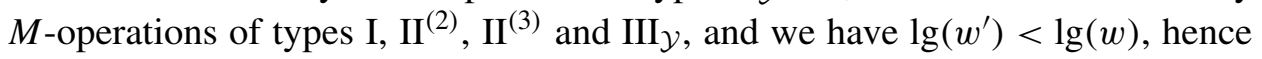
$w$ is not $M_{\mathcal{Y}}$-reduced.

Suppose that $w$ is not $M_{\mathcal{Y}}$-reduced. Let $w^{\prime}$ be an $M_{\mathcal{Y}}$-reduction of $w$, and let $g^{\prime}$ be the element of $\mathrm{KW}_{n}$ represented by $w^{\prime}$. Since $w^{\prime}$ is an $M_{\mathcal{Y}}$-reduction of $w$, the element $g^{\prime}$ lies in the coset $\mathrm{KW}_{n}(\mathcal{Y}) g$. Moreover, $\lg \left(g^{\prime}\right)=\lg \left(w^{\prime}\right)<\lg (w)=\lg (g)$, hence $g$ is not $\mathcal{Y}$-minimal.

Proof of Proposition 2.4. Let $\mathcal{Y}$ be a subset of $\mathcal{S}$. Consider the retraction $\rho_{\mathcal{Y}}$ : $\mathrm{KB}_{n} \rightarrow \mathrm{KB}_{n}(\mathcal{Y})$ constructed in Proposition 4.3. We shall prove that, if $u$ is a word over $\mathcal{S}^{ \pm 1}$, then $\overline{\pi_{\mathcal{Y}}(u)}=\rho_{\mathcal{Y}}(\bar{u})$. This will prove Proposition 2.4. Indeed, if $\bar{u}=\bar{v}$, then $\overline{\pi_{\mathcal{Y}}(u)}=\rho_{\mathcal{Y}}(\bar{u})=\rho_{\mathcal{Y}}(\bar{v})=\overline{\pi_{\mathcal{Y}}(v)}$. Moreover, since $\rho_{\mathcal{Y}}: \mathrm{KB}_{n} \rightarrow \mathrm{KB}_{n}(\mathcal{Y})$ is a retraction to the inclusion map $\mathrm{KB}_{n}(\mathcal{Y}) \hookrightarrow \mathrm{KB}_{n}$, we have $\rho_{\mathcal{Y}}(\bar{u})=\bar{u}$ if and only if $\bar{u} \in \mathrm{KB}_{n}(\mathcal{Y})$, hence $\overline{\pi_{\mathcal{Y}}(u)}=\bar{u}$ if and only if $\bar{u} \in \mathrm{KB}_{n}(\mathcal{Y})$.

Let $u=s_{1}^{\varepsilon_{1}} \cdots s_{l}^{\varepsilon_{l}}$ be a word over $\mathcal{S}^{ \pm 1}$. Let $\alpha$ be the element of $\mathrm{KB}_{n}$ represented by $u$.

- For $i \in\{0,1, \ldots, l\}$, we set $u_{i}^{+}=s_{1} \cdots s_{i}$, and we denote by $g_{i}$ the element of $\mathrm{KW}_{n}$ represented by $u_{i}^{+}$. 
- Let $i \in\{0,1, \ldots, l\}$. We write $g_{i}=h_{i} k_{i}$, where $h_{i} \in \mathrm{KW}_{n}(\mathcal{Y})$, and $k_{i}$ is $\mathcal{Y}$-minimal. Let $v_{i}^{+}$be an $M_{\mathcal{Y}}$-reduction of $u_{i}^{+}$. Then, by Lemma 4.4, $v_{i}^{+}$is a reduced expression of $k_{i}$.

- Let $i \in\{1, \ldots, l\}$. If $\varepsilon_{i}=1$, we set $z_{i}=k_{i-1} s_{i} k_{i-1}^{-1}$ and $w_{i}^{+}=v_{i-1}^{+} \cdot s_{i} \cdot$ op $\left(v_{i-1}^{+}\right)$. If $\varepsilon_{i}=-1$, we set $z_{i}=k_{i} s_{i} k_{i}^{-1}$ and $w_{i}^{+}=v_{i}^{+} \cdot s_{i} \cdot \operatorname{op}\left(v_{i}^{+}\right)$. Note that $w_{i}^{+}$is an expression of $z_{i}$.

- Let $i \in\{1, \ldots, l\}$. Let $r_{i}$ be an $M$-reduction of $w_{i}^{+}$. By Lemma 4.4, $r_{i}$ is a reduced expression of $z_{i}$. Note that we have $z_{i} \in \mathcal{Y}$ if and only if $r_{i}$ is of length 1 and $r_{i} \in \mathcal{Y}$.

- Let $i \in\{1, \ldots, l\}$. If $r_{i}$ is of length 1 and $r_{i} \in \mathcal{Y}$, we set $T_{i}=r_{i}^{\varepsilon_{i}}$. Otherwise we set $T_{i}=1$.

- By construction, we have $\hat{\rho}_{\mathcal{Y}}(\alpha)=\pi_{\mathcal{Y}}(u)=T_{1} T_{2} \cdots T_{l}$.

\section{Acknowledgment}

The research of Bellingeri was partially supported by the French government grant ANR-11-JS01-002-01.

\section{References}

[Artin 1947] E. Artin, “Theory of braids”, Ann. of Math. (2) 48 (1947), 101-126. MR 0019087 Zbl 0030.17703

[Bar-Natan and Dancso 2015] D. Bar-Natan and Z. Dancso, "Finite type invariants of w-knotted objects, I: w-knots and the Alexander polynomial", preprint, 2015. arXiv 1405.1956

[Bardakov 2005] V. G. Bardakov, "Virtual and welded links and their invariants", Sib. Èlektron. Mat. Izv. 2 (2005), 196-199. MR 2177995 Zbl 1100.57006

[Bardakov and Bellingeri 2009] V. G. Bardakov and P. Bellingeri, "Combinatorial properties of virtual braids”, Topology Appl. 156:6 (2009), 1071-1082. MR 2493369

[Bellingeri and Bodin 2016] P. Bellingeri and A. Bodin, "The braid group of a necklace", Math. Z. (online publication February 2016).

[Bourbaki 1968] N. Bourbaki, Éléments de mathématique, Fasc. XXXIV: Groupes et algèbres de Lie. Chapitre IV: Groupes de Coxeter et systèmes de Tits. Chapitre V: Groupes engendrés par des réflexions. Chapitre VI: Systèmes de racines, Actualités Scientifiques et Industrielles 1337, Hermann, Paris, 1968. MR 0240238 Zbl 0186.33001

[Charney and Paris 2014] R. Charney and L. Paris, "Convexity of parabolic subgroups in Artin groups", Bull. Lond. Math. Soc. 46:6 (2014), 1248-1255. MR 3291260 Zbl 1308.20037

[Chterental 2015] O. Chterental, "Virtual braids and virtual curve diagrams", J. Knot Theory Ramifications 24:13 (2015), art. ID \# 1541001. MR 3434540 Zbl 06532326

[Cisneros de la Cruz 2015] B. A. Cisneros de la Cruz, "Virtual braids from a topological viewpoint", J. Knot Theory Ramifications 24:6 (2015), art. ID \# 1550033. MR 3358444 Zbl 1327.57004

[Godelle and Paris 2012] E. Godelle and L. Paris, " $K(\pi, 1)$ and word problems for infinite type Artin-Tits groups, and applications to virtual braid groups”, Math. Z. 272:3-4 (2012), 1339-1364. MR 2995171 Zbl 1300.20045 
[Kamada 2007] S. Kamada, "Braid presentation of virtual knots and welded knots", Osaka J. Math. 44:2 (2007), 441-458. MR 2351010 Zbl 1147.57008

[Kauffman 1999] L. H. Kauffman, "Virtual knot theory", European J. Combin. 20:7 (1999), 663-690. MR 1721925 Zbl 0938.57006

[van der Lek 1983] H. van der Lek, The homotopy type of complex hyperplane complements, Ph.D. thesis, Radboud University Nijmegen, 1983.

[Manturov 2003] V. O. Manturov, "On the recognition of virtual braids", Zap. Nauchn. Sem. S.Peterburg. Otdel. Mat. Inst. Steklov. (POMI) 299:Geom. i Topol. 8 (2003), 267-286, 331-332. In Russian; translated in J. Math. Sci. 131:1 (2005), 5409-5419. MR 2038828 Zbl 1144.20310

[Serre 1977] J.-P. Serre, Arbres, amalgames, $\mathrm{SL}_{2}$, Astérisque 46, Société Mathématique de France, Paris, 1977. MR 0476875 Zbl 0369.20013

[Tits 1969] J. Tits, "Le problème des mots dans les groupes de Coxeter", pp. 175-185 in Symposia Mathematica (Rome, 1967/68), vol. 1, Academic Press, London, 1969. MR 0254129 Zbl 0206.03002

[Vershinin 2001] V. V. Vershinin, "On homology of virtual braids and Burau representation", J. Knot Theory Ramifications 10:5 (2001), 795-812. MR 1839703 Zbl 0997.57020

Received June 17, 2015. Revised February 18, 2016.

\section{PAOLO BELLINGERI}

Laboratoire Nicolas Oresme, ESPE de BAsse Normandie

UNIVERSITÉ DE CAEN

14032 CAEN

FRANCE

paolo.bellingeri@unicaen.fr

Bruno A. Cisneros de la CRUZ

InSTITUTO DE MATEMÁticAs DE LA UNAM

OAXACA DE JUÁREZ

OAXACA 68000

MEXICO

brunoc@matem.unam.mx

LUIS PARIS

IMB UMR5584, CNRS

Univ. Bourgogne Franche-Comté

21000 DIJON

FRANCE

lparis@u-bourgogne.fr 


\title{
PACIFIC JOURNAL OF MATHEMATICS
}

Founded in 1951 by E. F. Beckenbach (1906-1982) and F. Wolf (1904-1989)

$$
\text { msp.org/pjm }
$$

\section{EDITORS}

\author{
Don Blasius (Managing Editor) \\ Department of Mathematics \\ University of California \\ Los Angeles, CA 90095-1555 \\ blasius@math.ucla.edu
}

\author{
Paul Balmer \\ Department of Mathematics \\ University of California \\ Los Angeles, CA 90095-1555 \\ balmer@math.ucla.edu \\ Robert Finn \\ Department of Mathematics \\ Stanford University \\ Stanford, CA 94305-2125 \\ finn@math.stanford.edu \\ Sorin Popa \\ Department of Mathematics \\ University of California \\ Los Angeles, CA 90095-1555 \\ popa@math.ucla.edu
}

\author{
Vyjayanthi Chari \\ Department of Mathematics \\ University of California \\ Riverside, CA 92521-0135 \\ chari@math.ucr.edu \\ Kefeng Liu \\ Department of Mathematics \\ University of California \\ Los Angeles, CA 90095-1555 \\ liu@math.ucla.edu \\ Igor Pak \\ Department of Mathematics \\ University of California \\ Los Angeles, CA 90095-1555 \\ pak.pjm@gmail.com \\ Paul Yang \\ Department of Mathematics \\ Princeton University \\ Princeton NJ 08544-1000 \\ yang@math.princeton.edu
}

\section{PRODUCTION}

Silvio Levy, Scientific Editor, production@msp.org

\section{SUPPORTING INSTITUTIONS}

ACADEMIA SINICA, TAIPEI

CALIFORNIA INST. OF TECHNOLOGY

STANFORD UNIVERSITY

UNIV. OF BRITISH COLUMBIA

UNIV. OF CALIFORNIA, BERKELEY

UNIV. OF CALIFORNIA, DAVIS

UNIV. OF CALIFORNIA, LOS ANGELES

UNIV. OF CALIFORNIA, RIVERSIDE

UNIV. OF CALIFORNIA, SAN DIEGO

UNIV. OF CALIF., SANTA BARBARA
KEIO UNIVERSITY

MATH. SCIENCES RESEARCH INSTITUTE

NEW MEXICO STATE UNIV.

OREGON STATE UNIV.
Daryl Cooper

Department of Mathematics

University of California

Santa Barbara, CA 93106-3080 cooper@math.ucsb.edu

Jiang-Hua Lu

Department of Mathematics

The University of Hong Kong

Pokfulam Rd., Hong Kong

jhlu@maths.hku.hk

$$
\text { Jie Qing }
$$

Department of Mathematics

University of California

Santa Cruz, CA 95064

qing@ cats.ucsc.edu

\author{
UNIV. OF CALIF., SANTA CRUZ \\ UNIV. OF MONTANA \\ UNIV. OF OREGON \\ UNIV. OF SOUTHERN CALIFORNIA \\ UNIV. OF UTAH \\ UNIV. OF WASHINGTON \\ WASHINGTON STATE UNIVERSITY
}

These supporting institutions contribute to the cost of publication of this Journal, but they are not owners or publishers and have no responsibility for its contents or policies.

See inside back cover or msp.org/pjm for submission instructions.

The subscription price for 2016 is US $\$ 440 /$ year for the electronic version, and \$600/year for print and electronic.

Subscriptions, requests for back issues and changes of subscriber address should be sent to Pacific Journal of Mathematics, P.O. Box 4163, Berkeley, CA 94704-0163, U.S.A. The Pacific Journal of Mathematics is indexed by Mathematical Reviews, Zentralblatt MATH, PASCAL CNRS Index, Referativnyi Zhurnal, Current Mathematical Publications and Web of Knowledge (Science Citation Index).

The Pacific Journal of Mathematics (ISSN 0030-8730) at the University of California, c/o Department of Mathematics, 798 Evans Hall \#3840, Berkeley, CA 94720-3840, is published twelve times a year. Periodical rate postage paid at Berkeley, CA 94704, and additional mailing offices. POSTMASTER: send address changes to Pacific Journal of Mathematics, P.O. Box 4163, Berkeley, CA 94704-0163.

PJM peer review and production are managed by EditFLOW ${ }^{\circledR}$ from Mathematical Sciences Publishers.

PUBLISHED BY

\section{I. mathematical sciences publishers}

nonprofit scientific publishing

http://msp.org/

(C) 2016 Mathematical Sciences Publishers 


\section{PACIFIC JOURNAL OF MATHEMATICS}

Volume $283 \quad$ No. $2 \quad$ August 2016

The fundamental theorem of tropical differential algebraic geometry

FUENSANTA AROCA, CRISTHIAN GARAY and ZEINAB TOGHANI

A simple solution to the word problem for virtual braid groups

271

PaOlo Bellingeri, Bruno A. Cisneros de la CruZ and Luis

PARIS

Completely contractive projections on operator algebras

DAVID P. BLECHER and MATTHEW NEAL

Invariants of some compactified Picard modular surfaces and applications

AMIR DŽAMBIĆ

Radial limits of bounded nonparametric prescribed mean curvature surfaces

MOZHGAN (NORA) ENTEKHABI and KIRK E. LANCASTER

A remark on the Noetherian property of power series rings

BYUNG GYUN KANG and PHAN THANH TOAN

Curves with prescribed intersection with boundary divisors in moduli spaces of curves

\section{XIAO-LEI LIU}

Virtual rational Betti numbers of nilpotent-by-abelian groups

BEHROOZ MiRZAII and FATEMEH Y. MOKARI

A planar Sobolev extension theorem for piecewise linear homeomorphisms 405

EMANUELA RADICI

A combinatorial approach to Voiculescu's bi-free partial transforms

PAUL SKOUFRANIS

Vector bundle valued differential forms on $\mathbb{N} Q$-manifolds

LUCA VitAGLiANO

Discriminants and the monoid of quadratic rings 